\title{
Laparoscopic video analysis for training and image-guided surgery
}

\author{
PATRICIA SÁNCHEZ-GONZÁLEZ ${ }^{1,2}$, ALICIA M. CANO ${ }^{1}$, IGNACIO OROPESA ${ }^{1,2}$, \\ FRANCISCO M. SÁNCHEZ-MARGALLO ${ }^{3}$, FRANCISCO DEL POZO $^{1,2}$, PABLO LAMATA $^{1}$, \\ ENRIQUE J. GÓMEZ ${ }^{1,2}$
}

\author{
${ }^{1}$ Bivengineering and Telemedicine Centre (GBT), Technical University of Madrid (UPM), Madrid, Spain, ${ }^{2}$ Networking \\ Research Center on Bioengineering, Biomaterials and Nanomedicine (CIBER-BBN), Madrid, Spain, and ${ }^{3}$ Minimally \\ Invasive Surgery Centre fesús Usón (CCMIfU). Cáceres, Spain
}

\begin{abstract}
Automatic analysis of Minimally Invasive Surgical video has the potential to drive new solutions for alleviating needs of safe and reproducible training programs, objective and transparent evaluation systems and navigation tools to assist surgeons and improve parient safety. Surgical video is an always available source of informarion, which can be used without any additional incrusive hardware in the operaring room. This paper is focused on surgical video analysis methods and techniques. It describes auchors' contriburions in rwo key aspects, the 3D reconstruction of the surgical field and the segmentation and racking of tools and organs based on laparoscopic video images. Results are given to illustrate the potential of this field of research, like the calculi of the 3D position and orientation of a tool from its $2 \mathrm{D}$ image, or the translation of a preoperative resection plan into a hepatectorny surgical procedure using the shading information of the image. Research efforts are required to further develop these technologies in order to harness all the valuable informacion available in any video-based surgery.
\end{abstract}

Key words: $M I S$, video image analysis, $3 D$ reconsiruction, tool tracking, organ reconsiruction

\section{Introduction}

Minimally Invasive Surgery (MIS) is becoming a gold-standard choice for efficient, low-risk surgical procedures for several reasons. It is a safe, feasible, and patient-friendly way of performing a surgical intervention. Moreover, it satisfies patients' desire for faster recoveries and smaller injuries (aesthetic reasons), and shortens the hospitalization period. As a result, MIS is employed across various medical specialties and is replacing traditional surgical procedures as swiftly as technology and human adoption of change allow. Nevertheless, MIS presents important limitations for surgeons due to its new interaction paradigm compared to craditional open surgery. In MIS surgeons must navigate the anatomical landscape without the usual sensory clues. The deployment of this surgery is based on an indirect manipulation of specialized surgical tools introduced into the abdominal cavicy through small incisions. Surgeons follow the operation through an indirect visualization of the surgical field captured by an endoscope. The morivation of the analysis of MIS video is the development of solutions to tackle two basic clinical needs: Better training and objective evaluation of MIS skills, and an enhanced perception and interaction in the surgical field for creation of real-time navigation systems.

Acquisition of all the necessacy surgical skills requires a serious endeavour. Surgeons must follow long traineeship periods in order to become proficient in these techniques. Evaluation of surgical skills is needed for monitoring residents' leaming and for a transition towards a quality-based surgical culture. Current evaluation methods, based on the Halsted tradition, are in general subject to bias and halo effects. Steps have been taken to deal with these issues, such as the development of standardised

Correspondence: P. Sánchez-Gonzáles, Bioengineering and Telemedicine Centre (GBT), Technical University of Madrid (UPM), Avenida Complutense, 30, E-28040 Madrid, Spain, E-mail: psanchez@gbt.tfo.upm.es 
questionnaires as objective structured assessment of technical skill (OSATS) and their MIS counterpart, global operative assessment of laparoscopic skills (GOALS) (1). Nevertheless, there is a shared opinion that new training and evaluation programs are required (2) in order to obtain skills outside the operating room (OR) and certify progress accordingly. These programs have in common the need for an objective evaluation of the surgical skills, regardless of the methodology and systems employed. Those new assessment methodologies should be transparent for the trainee, not requiring direct supervision and providing immediate feedback.

There are two main research issues related to providing an objective evaluation of surgical skills: The definition of meaningful evaluation metrics and the development and adoption of non-intrusive acquisition systems. The first one has generated an interesting debate over the last few years on which metrics discriminate surgical performance better (3). Two main categories are proposed (4): Efficiency metrics based on physical data such as movements and forces applied, and quality metrics concerned with the overall performance and the errors incurred. Acquisition systems have evolved in parallel following two different courses: Sensorbased systems and virtual simulation. Sensor-based systems are employed in controlled environments such as box trainers to capture physical information regarding the task at hand. This information is usually based on the forces applied (5) and/or the kinematics involved (6), which can be employed for speed and acceleration calculi, optimal path and economy of movement determination, depth perception and movement sequences analysis, and repetitions or idle states detection. Virtual reality simulators are also an always available training workbench for the resident and surgeon, which can not only emulate the laparoscopic interaction, but also act as a virtual instructor that guides the trainee and provides him with constructive feedback (7).
On the other hand, surgical navigation systems are providing new possibilities for MIS. These systems allow to transfer preoperative data, images and decisions to the OR (Figure 1a and b), and give the surgeon guidance during the procedure (Figure 1c). Traditionally, the surgeon had to translate all this information mentally to the real scenario in front of him, with the difficulties this entails, most notably disorientation with respect to the medical image studies. Image-guided surgery (IGS) systems have the potential to compensate these limitations. However, difficulties arise in the case of soft-tissue surgeries, such as those of the abdominal cavities, where there are technical challenges to cope with such as tissue deformation, shifting and other topological changes caused by the pneumoperitoneum, respiration, heart rate or tissue manipulation (8). Thus, organ tracking presents even greater difficulties due to said phenomena, and the use of fiducial markers and optical or electromagnetic systems to track organ deformations is not a feasible solution in many cases. Usually, location and tracking of organ position and deformation are addressed by the use of intra-operative medical imaging modalities (most notably US, CT and MR) (9) and some specialised hardware based on laser or structured light technology $(10,11)$. Tools' tracking is also a critical issue in any IGS solution, since optical and electromagnetic systems are the most widely used in the OR. However, they both add extra instrumentation in the OR, which can disturb surgeons during interventions (12).

Automatic analysis of surgical video sequences has applications in surgical training, evaluation and navigation systems (13). It provides useful information about the position of instruments and organs, surgical manoeuvres, measurements of distances or even an approximate $3 \mathrm{D}$ reconstruction of the surgical scene. Laparoscopic video images are an always available source of information and can be used without adding extra technological components in the OR. Moreover, with the development of high definition endoscopes

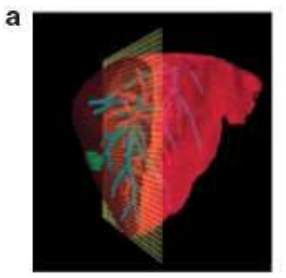

b

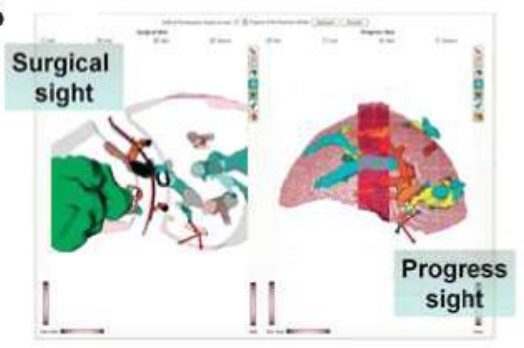

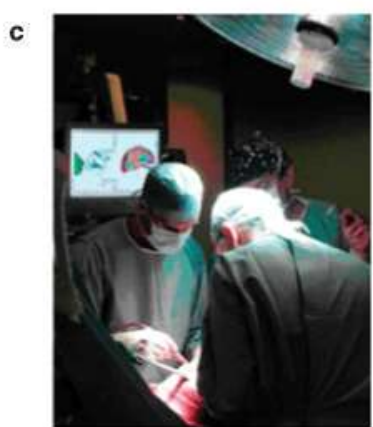

Figure 1. Example of an image-guided surgical system: (a) Liver virtual model; (b) snapshot of the navigation system with the surgical and progress sight and (c) intra-operative use of the system. 
and monitors, this informacion is sharper and cleaner than ever, and its analysis is surely to benefit from these crends. Following sections illuscrate the potential of harnessing laparoscopic video images in order to improve surgical training, evaluation and guidance.

\section{Laparoscopic video analysis: Literature overview}

In order to increase pacient safety during laparoscopic surgery, rraining systems can include overlays with tool rracking informacion, positions of anatomical structures and direccions to follow in the surgical scenario. Immediate visual feedback can be obrained from training systems to help surgeons during their formation, monitoring and evaluating the entire process to minimize potential hazards, identify problem areas and find solutions. In training systems, cognitive skills training can benefit from video analysis by converting surgical videos into multimedia didactic contents adding texts, images, 3D virtual animations, etc. For these applicarions, segmentacion and tracking are required to idenuify organs, regions of interest or areas where special attention should be paid during the surgical intervencion, and warn about the proximity of delicate regions.

Multiple semiautomatic segmentation methods can be found in the literature, based e.g. on morphological border detection combined with growing region techniques (14), or in a combination of a growing segmentation and image enhancement steps based on the Bezier model (15). Moreover, some automatic segmentacion approximations can be found, such as an algorithm based on a vague initial graph-based segmentacion and a posterior merging multiregion stage, where a modificarion of the Fischer criteria is used (16).

Tool segmentation and tracking constitute another important contribution of video analysis. Keeping surgical tools centred in the field of view of the image is most important in MIS in order to reduce risks of damaging non-visible anatomical structures. Segmentacion has been addressed using several approaches, using colour either on red, green, and blue (RGB) $(17,18)$ or hue, saturacion value (HSV) $(19)$, space, geometrical properties such as border detection (20), or a combination of both (21).

Acquisition of the 3D shape of the surgical scenario has a wide variecy of applications, especially in the field of minimally invasive surgery. There is a wide range of computer vision techniques for this purpose, and shading, stereoscopy and motion are the three main physical cues studied so far in a MIS video. Shading refers to the light intensity reflected by a physical object dependent on the geometrical relation between its surface and the source of light. Shape from shading reconstruction techniques exploit this physical property of the behaviour of light in the surgical scene (22). Shape from stereo vision refers to the abilicy to acquire information on the $3 \mathrm{D}$ structure and the relative distances on the scene from two or more intensity images taken from different viewpoints $(23,24)$. However, they are not always feasible in MIS applications because stereoscopic endoscopes are not generally available in hospitals. Structure from mocion techniques obtain depth information through movement analysis (25-27). These algorithms usually compute local frame-to-frame motion feature matching and are refined in a global optimization moving backward and forward through the whole sequence (called bundle adjustment). State-of-the-art technology solves this problem in a scenario with staric objects or rigid movements. In MIS these solutions have therefore limited application because movements of anatomical scructures are non-rigid. An interesting approximation uses simulataneous localization and mapping (SLAM) technology (28) which extracts in real-time not only the $3 \mathrm{D}$ position of some key landmarks, but also the 3D trajectory of a monocular camera moving rapidly through a previously unknown scene.

As briefly described here, there have been recent advances in video image processing and computer vision algorithms in order to enrich information provided by laparoscopic surgical videos. In this paper we focus on wo main applications of video analysis: Non-intrusive tracking of tools and ongans, and 3D reconstruction of the surgical scene.

\section{Non-intrusive tracking of organs and tools}

Traditionally, object tracking is achieved by devices based on mechanical, optical, acoustic or electromagnetic technologies (29). Their introduction in the OR is a key limiting factor, since they disturb the surgical workflow and ergonomics, which may compromise parient safery. Surgical video analysis provides a nonincrusive alternarive for rracking organs and tools in the OR. This solucion requires two main steps, first to find the $3 \mathrm{D}$ position and orientation of the surgical camera in the OR, and second to locate the organs and tools with respect to the optical centre of the camera. This section describes methods and illustrates results of the second step of this methodology, formulating a simplified scenario with a known and fixed camera locacion. 


\section{Organs' tracking}

The main problem to be solved when tracking a structure in video sequences is the identification of the $2 \mathrm{D}$ position and area of said structure. This is also a key issue in object recognition, scene and image understanding. Segmentation algorithms are thus needed not only for organ tracking, but also as a preliminary step for a $3 \mathrm{D}$ reconstruction of shape or for adding or enhancing information present in the surgical scene. These algorithms exploit different image properties or organs and anatomical structures, such as colour, textures, edges or shading.

Characteristics of the segmentation algorithm developed for laparoscopic images will be constrained by its potential application. Thus, a manual or semiautomatic algorithm will be sufficient for augmentation of videos for surgical training. However, for IGS applications the segmentation process needs to be automatic and real-time. Laparoscopic images have both small and large anatomical structures, which adds an additional level of difficulty for an automatic algorithm. It has to be noted that a segmentation of structures is mostly limited to those which are clearly discernible in the image by the algorithm, as e.g. vessels by use of a contrast agent (8). The segmentation algorithm used for laparoscopic images has to be able to detect any region that has a slightly different colour compared to its neighbouring regions, because small differences in colour or texture may indicate different or special regions that should be taken into account when performing a surgical technique. Laparoscopic sequences have features that make segmentation quite challenging, like the lack of sharp borders, specularities, interreflections and colour variability due to clinical condition.

In contrast with traditional approaches to the problem, we propose in this paper a method which exploits shading as an additional segmentation criterion. A split and merge technique is chosen as the evolution strategy in the method. The method is based on a simplified illumination model with light source and camera systems located in the same spatial point (30). An illumination equation enables the definition of an optimal feature to distinguish structures, which is the gradient of the logarithm of image intensity. The logarithm simplifies the segmentation process when constructing the gradient image by making an homogenisation in the region of a structure. The gradient of the logarithm reaches its maximum in the edge of a region, which is suitable for a combination of watershed segmentation (split step) and a merging step with Hotelling's T-square (or alternatively Mahalanobis distance) as the similarity metric. An exemplary result of this method is illustrated in Figure 2, where 2a shows the original image and $2 \mathrm{~b}$ the segmented image. Note how the method is able to segment both large organs, i.e. liver or spleen, and anatomical details such as vessels.

\section{Tools' tracking}

Automatic video analysis provides different solutions to detect the $2 \mathrm{D}$ or $3 \mathrm{D}$ position, and even orientation, of surgical instruments during a surgical process. Some applications will only require to follow a characteristic $2 \mathrm{D}$ point of the tool, as for example its tip. For IGS applications, real-time, accurate and robust tracking of tools during the entire surgical procedure is required. Nevertheless, these conditions can be relaxed in an off-line analysis of a video sequence for objective evaluation. Video-based tool tracking must cope with partial occlusions, the insertion and removal of different tools, and with image quality degradation caused by gas and smoke. Our approach solves the problem of calculating the $3 \mathrm{D}$ position and orientation of a tool with only the $2 \mathrm{D}$ information extracted from each frame of a video sequence. Two pre-processing steps are needed, distortion correction and segmentation, before the application of the mathematical solution for it.

Endoscopic images are affected by image distortions, mainly geometrical deformations as vignetting
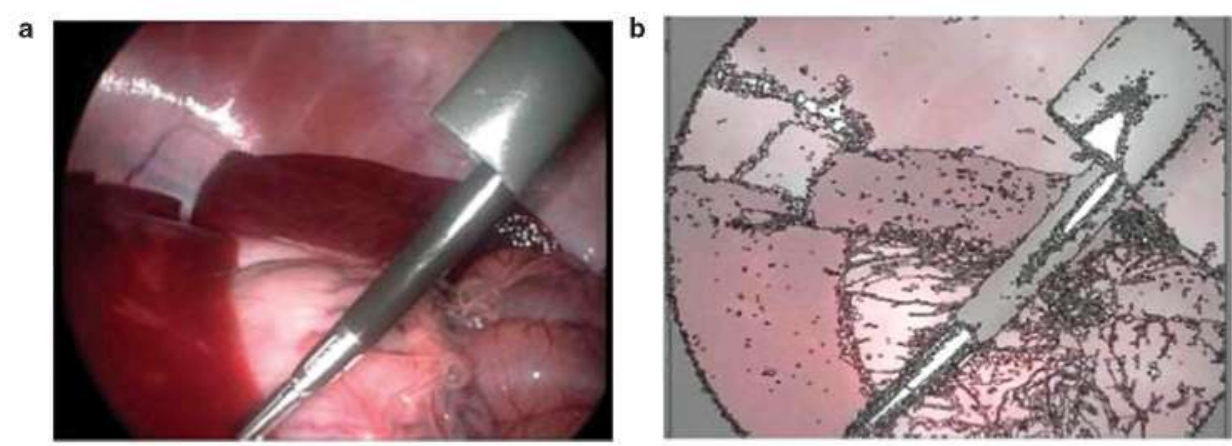

Figure 2. (a) Original image, (b) automatic segmentation results. 
and barrel distortion (also named eye globe distortion), which cause straight lines to appear in the image as curves ones. A barrel distortion correction method uses a calibration procedure in order to define a rectification mapping function. The idea is to capture an image of a regular pattern drawn in a board in order to characterise the distortion. The rectification geometrical transformation is built finding the deformation between the captured image and its homologous corrected one. This is illustrated in Figure 3 (3a represents the original frame and $3 b$ the result of the distortion correction process) (31).

A segmentation algorithm is then used to detect surgical tools present in the surgical image. The algorithm exploits two key tools characteristics:

- surgical tools exhibit rigid motion;

- tools edges can be approximated by straight lines.

The algorithm takes two steps (Figure $4-4 \mathrm{a}$ is the original image), the detection of regions of interest (ROIs) (Figure 4b), and the detection of edges of tools (Figure 4c). ROIs are defined as the area which includes the tool black axis. An analysis of both colour components and geometrical properties such as orientation and size are used for this ROIs identification. The result is a simplified image where anatomical structures are deleted, as well as the metallic tool tip (Figure $4 \mathrm{~b}$ ). The detection of edges is achieved by combination of a laplacian gradient filter and Hough or Radon transforms. Finally, knowing the tool's cylindrical geometrical dimensions and its $2 \mathrm{D}$ projection as denoted by the detected borders, real 3D pose of tools is calculated. The mathematical equation for this calculus is a description of the geometrical relation between the tools, trocars and the optical centre of the camera, and its complete description and explanation can be found in (31). Tool tip localization Root Mean Square error (in $Z$ coordinates) has been characterised as $9.28 \mathrm{mmRMS}$, and the performance is dependent on the quality of the image of the tools (32). This tracking performance is good enough for gesture analysis and objective evaluation

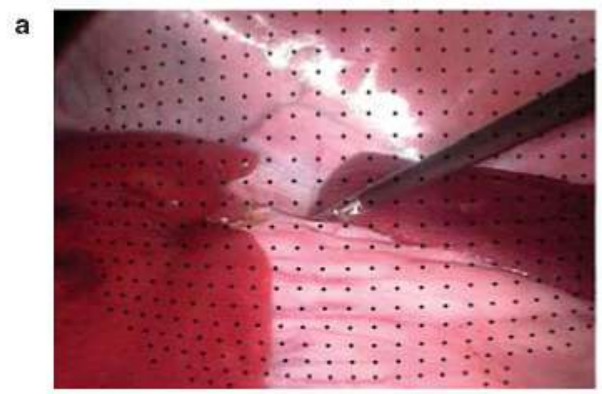

Figure 3. (a) Original frame, (b) result of distortion correction. of surgical manoeuvres. However, IGS applications require higher accuracy.

\section{D reconstruction of a surgical field from shading}

A 3D reconstruction allows visualizing anatomy from different angles, which can enhance the appreciation of the surgical scene. Characteristics of the reconstruction of a 3D model from laparoscopic images will be strongly affected by its application, and its desired accuracy, robustness, velocity or computing cost will be constrained by this issue. For example, on a training scenario, where videos are pre-recorded and there is no real time limitation, conditions will be more lenient than on an IGS one. The handling of laparoscopic image data in IGS requires realtime algorithms and also careful synchronization; in particular between simultaneously acquired data from heterogeneous sources (the registration problem). Accuracy and robustness needed in this case scenario are extremely important due to risks carried out when operating a patient in the OR. Moreover, accurate methods have to be developed to register image space to physical or patient space based on video processing.

We here address the reconstruction problem using shading information. As explained before, shape from shading (SfS) techniques amount to $3 \mathrm{D}$ reconstructing a surface from an image captured by the endoscope, given surface, light source and camera parameters. Although shading can be in a first analysis a poor approach, there are several reasons for using it:

(1) It is an interesting new approach, and

(2) although it implies some difficulties in laparoscopic scenarios light source is controlled and fixed in its optical centre.

Two methods have been implemented for calculating a depth map of the surgical scene, assuming a lambertian light interaction model with the surface. It is important to realise that by using shading information alone it is not possible to determine the absolute depth of the whole surgical scene. This limitation

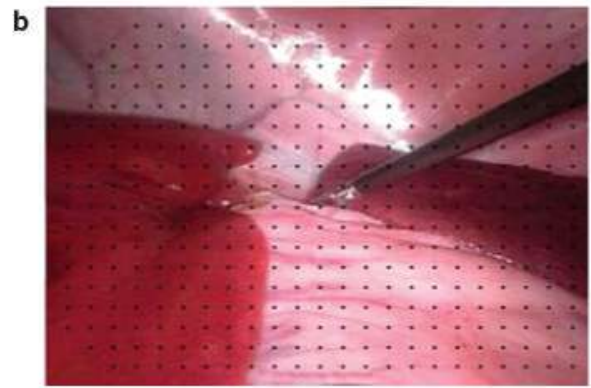



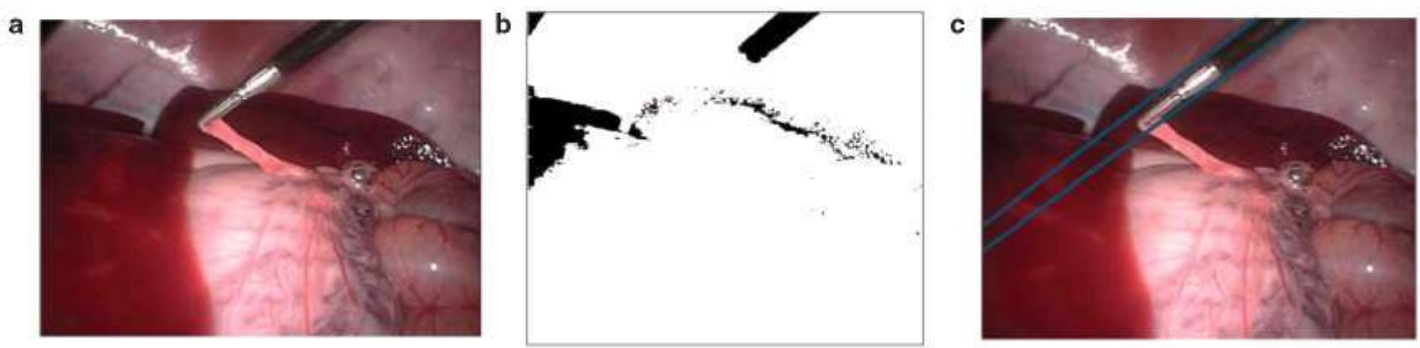

Figure 4. Process to calculate the 3D position and orientation of a surgical tool in a laparoscopic photogram: (a) Original frame, (b) result of ROI identification and (c) detection of edges.

leads to a scale ambiguity problem. Knowing this limitation, the purpose of the analysis of one frame should be to achieve the relative depth map associated to the laparoscopic image, which is the objective of the first method. The second method described here corrects for scale uncertainty by employing preoperative knowledge of the shape under study.

The first method proposes an algorithm based on a simplified illumination model of the surgical scene (29). The relative depth map is obtained by computing the image intensity gradient in a given direction. The $3 \mathrm{D}$ reconstruction is then calculated adding all the differential increments of depth and dividing the gradient by the image intensity. An exemplary result of this method is shown in Figure 5, illustrating the original image captured by the endoscope during the surgical intervention (Figure 5a) and the depth map associated to it, as well as the $3 \mathrm{D}$ reconstruction combining scene and tool reconstruction. Results in Figure $5 \mathrm{~b}$ show how shines present in a

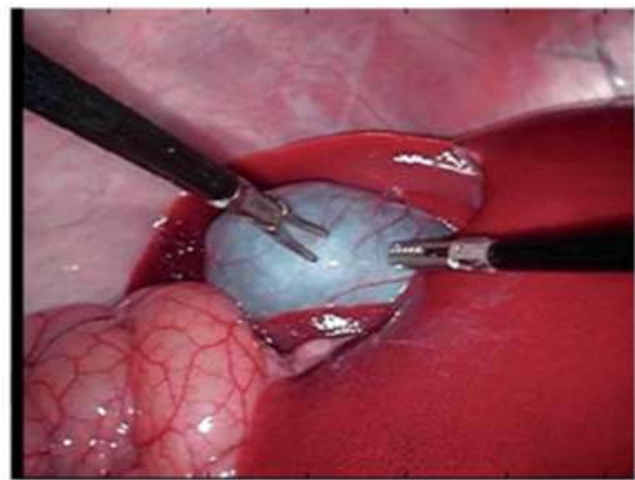

b

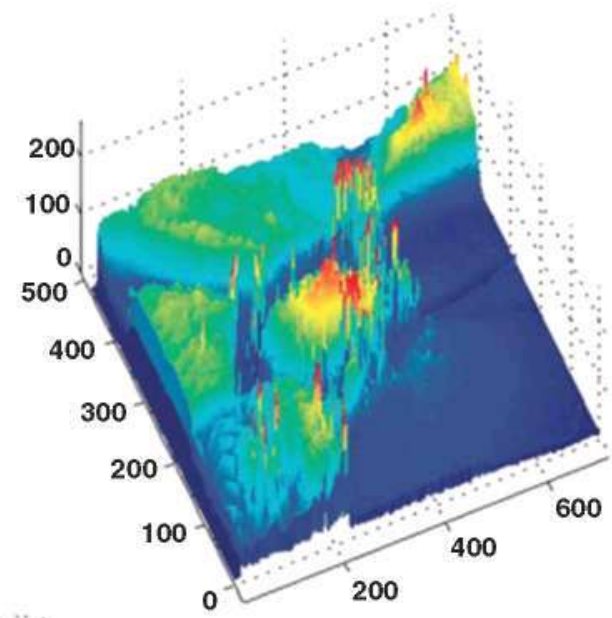

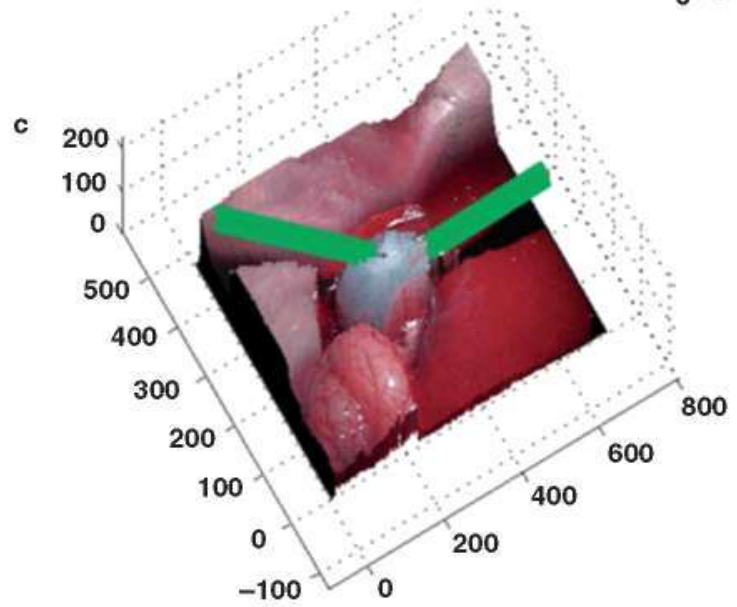

Figure 5. (a) Original laparoscopic image, (b) depth map, (c) image 3D reconstruction combining scene reconstruction and tool localization (green cylinders). 
the image limit the method resolution. Thus, to cancel their effects a pre-processing step is required. However, since the scope of this paper is to show the virtues of the $3 \mathrm{D}$ reconstruction method, its influence is omitted on the results given in Figure 5b. Their effects have been corrected in Figure 5c.

The second method uses a propagation SfS technique initialized from the closest point to the camera (33). Its development is specifically motivated by augmentation of laparoscopic hepatectomy and aims at integrating visualization of major vessels and targeted tumours as well as preoperative resection planning information into the endoscopic images (Figure 6). It takes into account the assumption that the closest point in the image is the brightest, which is valid for the geometry of the liver. It then performs an iterative $3 \mathrm{D}$ reconstruction and $3 \mathrm{D}$ registration via a modified ICP (Iterative Closest Point algorithm) to compensate for unknown scale and a correction of an unknown albedo and changing lighting conditions.

An important limitation of shading information is the presence of noise sources, such as reflections or specularities. These reflections originate in surrounding organs, and are quite unpredictable and variable due to movements caused by respiration, cardiac motion, and mechanical shift accompanying the surgical intervention. Our current work is focussed on correcting non-linearities in the acquisition of images and nonhomogeneities in the radiance of the source of light.

\section{Discussion}

Video image analysis and computer vision algorithms have had a great impact in the last decades applied on fields such as surveillance, control process or the entertainment business. Thus, it is our belief that such a potential source of information will prove invaluable in minimally invasive surgery, where it is an ever-present resource. In itself, video information is quite intuitive to the human eye. Still, there are several aspects which can be difficult to process mentally and which a computer can handle much better, such as an automatic extraction of the $3 \mathrm{D}$ orientation of a surgical tool.

Video image analysis can tackle some human perceptual limitations and improve surgeons' awareness. But, unlike the human eye and brain, no algorithm is completely general, i.e. able to perform its intended function given any possible video input. Thus, it is critically dependent on certain unique assumptions about the real-world video scene it is expected to analyze. Without these assumptions, methods can produce ambiguous results. Thus, a relevant stage in the solution development process is modelling the surgical scene and the imaging system. The final application clearly conditions requirements.

Surgical video analysis provides an alternative sensing strategy to track tools and organs. An important advantage over sensor-based systems, optical or electromagnetic (EM) tracking technologies, is that this solution does not require any additional hardware, and, compared to EM sensing, is immune to background EM noise. Sensors also deteriorate ergonomics of surgical instruments and thus change the way the surgeon holds them or experiences their form and weight. Video analysis is already the technology used in some hybrid surgical simulators for tool tracking (34).

Video-based tracking of surgical tools allows the registration of objective surgical evaluation metrics

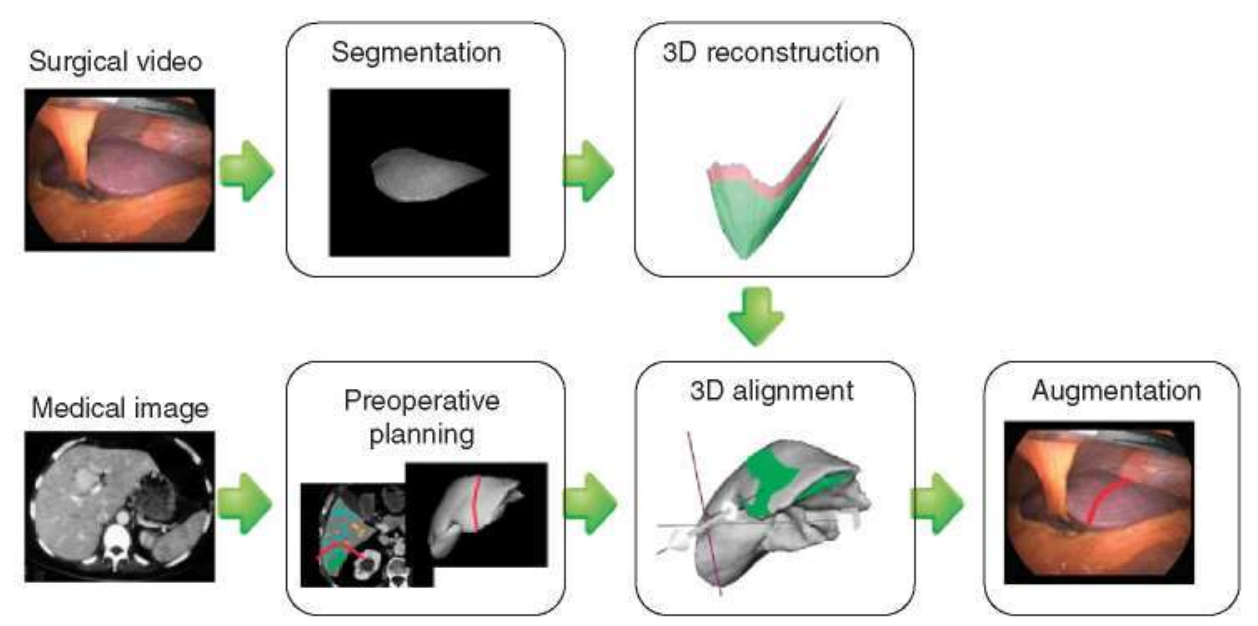

Figure 6. Data pipeline for the augmentation of the surgical view in a liver resection procedure, where the resection line decided in a preoperative stage is overlaid on the surgical image. It is based on a 3D reconstruction of the liver using a shape from shading propagation technique, and a $3 \mathrm{D}$ alignment which compensates for the scale and albedo ambiguity. 
not only on controlled settings such as box crainers, but also in the OR. The information about kinematics, regarding their posicion and crajectories, is available straightaway, and this serves as the grounds to extract higher levels of evaluation with activity recognition algorithms (35). Useful information can then be extracted about the surgical steps taken, mainly related to the order of performance, repetitions and idle states. Although such analyses can be done manually (36), this is a tedious, rime-consuming task. Automarion of this task is achieved by manoeuvre video-based recognicion systems. Moreover, a 3D reconstruction of the surgical scenario would allow to measure distances berween inscruments and tangets or landmarks previously established, which can also be used for evaluation purposes.

In the field of IGS, endoscopic video analysis can be a valuable information source, as a means of tracking both organs and laparoscopic tools. Tracking systems are critical for computer-assisted image-guided intervencions: They provide the necessary real-ime informacion on the localization of equipment (mainly surgical tools) and parients. Some efforts have been done using video in image guided applications for hepatic (37) and urologic (38) laparoscopic surgeries. Nevertheless, a video-based system is limited by image qualicy and field of view, and in this field of IGS this technology will probably complement, and not replace, ocher solutions.

Another relevant application of video analysis is superimposing pre-operatory informarion, like virtual anatomical reconstructions extracted from medical images, over surgical images, offering a view in transparency of the parient on the video images (39). One important strength of a video-based solution is its potentially higher accuracy, based on the fact that the alignment berween the real and virtual anatomy of the patient is calculated from the same image where the information is superimposed. Some authors suggest that image-guided systems will be simplified if automated image registracion is developed, which will need a real-cime video analysis tool (40). However, there are important challenges to be tackled such as adaptacion to the variability of qualicy and appearance of images, strongly affected by surgical accions and also by inter-parient biological variability.

There are also some limitations to be considered regarding the information extracted from a video sequence. Information at any given moment will be constrained by the camera's field of view. Indeed, any possible real-time adjustments regarding the tool and ongan position and orientation will be limited by the field of view of the endoscope. Also, robust solutions are needed for tracking the camera shifting due to incra-operacive movements and field of view adjustments. This issue has not been tackled in this paper, but camera tracking solucions like the MonoSLAM (28) are necessary in order to obtain fullyequipped navigation systems.

Our long-term vision is to achieve robust and instantaneous automatic video analysis algorithms, and there are important challenges in front of the scientific communicy. Thus, in order to achieve a quick and effecrive 3D reconstruccion, an oprimal combination of shading, motion and texture should be found, exploicing all cues present in endoscopic video images. Moreover, an important issue is the design of a complete tracking system, fusing endoscope positioning system with a robust organ and tool tracking solution. Furthermore, video-based solutions can be combined wich other hardware-based algorithms for precision increase.

Nowadays, the more challenging aspects are concentrated in IGS applications, where accuracy and real-cime needs are crucial issues. On the orher hand, these constraints are laxed for evaluation purposes, where data analysis can be carried out offline and precision is not so critical. To date, real-cime conditions for IGS applications have not been achieved. Surgical evaluation applications need complete and accurate task analysis of all operating techniques. It is not an easy task because it requires breaking down a surgical procedure into sub-tasks thanks to a rigorous observation process with expert surgeons.

\section{Conclusion}

Automatic analysis of MIS video has the potential to drive new solutions for alleviaring needs of safe and reproducible training programs, objeccive and rransparent evaluacion systems and navigacion systems to assist surgeons and improve parient safery. This source of informarion is always available and can be used without any additional intrusive hardware. This paper has shown new methods for tracking and 3D reconstruction, and has illustrated some of the current challenges to be solved in order to harness all its potential.

Declaration of interest: The authors report no conflicts of interest. The auchors alone are responsible for the content and writing of the paper.

\section{References}

1. Moorhy K, Munz Y, Sarker SK, Darzi A. Objective assessment of technical skills in surgery. BMJ: British Medical Joumal 2003;327:1032.

2. Feldman LS, Sherman V, Fried GM. Using simulators to assess laparoscopic competence: ready for widespread use? Surgery $2004 ; 135: 28-42$. 
3. Satava RM, Cuschieri A, Hamdorf J. Merrics for objective assessment. Surg.Endosc. 2003;17:220-6.

4. Fried GM, Feldman LS. Objective Assessment of Technical Performance. World joumal of surgery 2008;32:156-60.

5. Rosen J, Brown JD, Chang $L$, Barreca $M$, Sinanan $M$, Hannaford B. The BlueDragon-a system for measuring the kinematics and the dynamics of minimally invasive surgical tools in-vivo. Proceedings- IEEE Int Conf on Roborics and Automacion 2002:1876-81.

6. Chmarra MK. TrEndo Tracking System. Motion Analysis in Minimally Invasive Surgery. PhD. 2009.

7. Lamaca P, Gómez EJ, Bello F, Kneebone RL, Aggarwal R, Lamara F. Conceptual framework for laparoscopic VR simulators. IEEE Comput Graph Appl. 2006;26:69-79.

8. Baumhauer $M$, Feuerstein $M$, Meinzer HP, Rassweiler $J$. Navigarion in Endoscopic Soft Tissue Surgery - Perspecrives and Limicacions. Joumal of Endourology 2008;22: 751-66.

9. Marvik $R$, Lango $T$, Tanguen $G A$, Iindseth $F$, Yavuz $Y$, Nagelhus Hemes TA. Image-guided laparoscopic surgery. Review and current suatus. Minerva Chir. 2005;60: 305-25.

10. Cash DM, Miga MI, Glasgow SC, Dawant BM, Clemens $\mathbf{L W}$, Cao $\mathbf{Z}$, et al. Conceps and preliminary data toward the realization of image-guided liver surgery. J Gastrointest Surg. 2007;11:844-59.

11. Ackennan J, Keller K, Fuchs H. Surface Reconstruction of Abdominal Organs Using Laparoscopic Structured Light for Augmented Realiry Applications. Proceedings of SPIE 2002; 4661:39-46.

12. Lamata P, Lamata F, Sojar V, Makowski P, Massoptier Lo Casciaro $\mathrm{S}$, et al. Use of the Resection Map system as guidance during hepacectomy. Surg Endosc. 2010 Feb 23. [Epub ahead of print].

13. Sánchez-González P, Oropesa I, Cano AM, Gayá F, Lamata P, Sánchez-Margallo FM, et al. Endoscopic video images analysis for surgical training and image-guided surgery, World Congress on Medical Physics and Biomedical Engineering (WC2009); IFMBE Proceedings 2009:25:251-4.

14. Hsiao YT, Chuang CL, Jiang JA, Chien CC. Robust multiple objects tracking using image segmentacion and trajectory escimacion scheme in video frames. Image and Vision Computing 2006;24:1123-36.

15. Li Y, Lu D, Lu X, Liu J. Interactive color inage segmentation by region growing combined with Image enhancement based on bezier model. Proceedings of the Third Intemational Conference on Image and Graphics (ICIG'04) 2004:96-9.

16. Shu Y, Bilodeau GA, Cheriet F. Segmentation of Laparoscopic Images: Integrating Graph-Based Segmentarion and Mulcistage Region Merging. Proceedings of the Second Canadian Conference on Computer and Robot Vision (CRV'05) 2005:429-36.

17. Voros S, Long JA, Cinquin P. Automaric localization of laparoscopic instruments for the visual servoing of an endoscopic camera holder. Med. Image Comput. Comput. Assist. Interv. 2006;9:535-42.

18. Casals A, Amat J, Laporte E. Automatic guidance of an assistant robot in laparoscopic surgery. Proceedings 1996 IEEE International Conference on Robotics and Automaiion 1996;1:895-900.

19. Krupa A, Gangloff J, Doignon C, de Mathelin M, Morel G, Leroy $\mathrm{J}$, et al. Autonomous 3-D posicioning of surgical instruments in robotized laparoscopic surgery using visual servoing. IEEE Transactions On Robouics And Automation - Special Issue On Medical Roborics 2003;19:842-53.
20. Tonet $O$, Ramesh TU, Megali $G$, Dario P. Tracking endoscopic instruments without localizer: image analysis-based approach. Stud. Health Technol Inform. 2006;119:544-9.

21. McKenna SJ, Nait Chanif H, Frank T. Towards Video Understanding of Laparoscopic Surgery: Instrument Tracking. Conf. Image and Vision Computing New Zealand 2005: $317-21$.

22. Zhang $R$, Tsai PS, Cryer $\mathbb{J}$, Shah $M$. Shape from Shading: A Survey. IEEE Transactions on pattern analysis and machine intelligence 1999;21:690-706.

23. Samaras D, Metaxas D, Fua P, Leclerc YG. Variable Albedo Surface Reconstrucrion from Stereo and Shape from Shading. IEEE Computer Society Conference on Computer Vision and Pattern Recognition (CVPR'00) - 2000;1:1480.

24. Banks J, Bennamoun $M$, Kubik K, Corke P. An accurate and reliable stereo matching algorithm incorporaring the rank constraint Symposium on Intelligent roboric systems1999; 23-32.

25. Newman PM. On the structure and solution of the simultaneous localizacion and map building problem. Ph.D. dissercacion, University of Sydney, 1999.

26. Jebara T, Azarbayejani A, Pentland A. 3D Structure from 2D Motion. IEEE Signal Processing Magazine 1999;16:66-84.

27. Dellaert F, Seiz S, Thorpe C, Thrun S. Structure from Motion without Correspondence. IEEE Computer Society Conference on Computer Vision and Pattern Recognition (CVPR'00), 2000.

28. Davison AJ, Reid I, Molton $\mathrm{N}$, Stasse $\mathrm{O}$. Real-time single camera SLAM. IEEE Trans. Patrem Anal.Mach. Intell. 2007; 29:1052-67.

29. Chmarra MK, Grimbergen CA, Dankelman J. Systems for uracking minimally invasive surgical instruments. Min Invas Ther and Allied Technol. 2007;16:328-40.

30. Sánchez-González P, Gayá F, Cano AM, Gómez EJ. Segmentation and 3D reconstruction approaches for the design of laparoscopic augmented reality environments. Lecture Notes in Computer Science (LNCS) 2008:127-34.

31. Cano AM, Vara I, Sánchez-González P, Gómez EJ. Laparoscopic image analysis for automatic tracking of surgical tools. Proceedings of Computer Assisted Radiology and Surgery (CARS 2008), 2008;3:S279.

32. Cano AM, Gayá $F$, Lamaca $P$, Sánchez-González $P$, Gómez EJ. Laparoscopic tool tracking method for augmented reality surgical applications, Lecure Notes in Computer Science LNCS 2008;5104:191-6.

33. Lamata $P$, Morvan $T$, Reimers $M$, Samset $E$, Declerck J. Addressing Shading-Based Laparoscopic Regisuration. World Congress on Medical Physics and Biomedical Engineering 2009:189-92.

34. ProMIS simulawr: http $/ /$ www.haptica.com/promis.hrm. Last access: 15th April.

35. Estebanez B, Jimenez G, Muñoz V, Garcia-Morales I, Bauzano E, Molina J. Minimally Invasive Surgery Maneuver Recognition Based on Surgeon's Model. 2009 IEEE/RSJ International Conference on Intelligent Robots and Systems, 2009.

36. Rosen J, Brown $\mathrm{D}$, Chang $\mathrm{L}$, Sinanan $\mathrm{MN}$, Hannaford $\mathrm{B}$. Generalized approach for modeling minimally invasive surgery as a stochastic process using a discrete Markov model. Biomedical Engineering, IEEE Transactions 2006:53:399-413.

37. Herline A, Stefansic JD, Debelak J, Galloway RL, Chapman WC. Technical advances toward interacrive image-guided laparoscopic surgery. Surg Endosc. 2000;14:675-9.

38. Teber D, Guven S, Simpfendo T, Baumhauer M, Güven EO, Yencilek F, et al. Augmented Reality: A New Tool To 
Improve Surgical Accuracy during Laparoscopic Parrial Nephrectony? Preliminary In Viuro and In Vivo Results. European Urology 2009;56:332-8.

39. Soler $L$, Ayache $N$, Nicolau $S$, Pennec $X$, Forest $C$, Delingette $\mathrm{H}$, et al.. Virtual Realiry, Augmented Reality and Robotics in surgical procedures of the liver. Perspectives in
Image-guided Surgery. Proceedings of the Scientific Workshop on Medical Robotics, Navigacion and Visualization (MRNV) 2004:476-84.

40. Marescaux J, Rubino $F$, Arenas $M$, Mutter D, Soler L. Augmenced Reality-Assisced Laparoscopic Adrenalectomy. JAMA 2004;18:2214-5. 